\title{
Die soziale Distanz von polnischen Auswanderern zu Fremden in einem neuen soziokulturellen Umfeld
}

Streszczenie: Niniejszy artykuł jest próbą opisania zjawiska najnowszych migracji Polaków, które mają miejsce po 2004 roku, kiedy Polska stała się państwem członkowskim Unii Europejskiej i kiedy wielu Polaków znalazło zatrudnienie za granicami własnego kraju. Większość migrantów z Polski, relatywnie dobrze wykształconych, młodych i mających przykre doświadczenia w kraju wysyłającym, radzi sobie w nowych okolicznościach społecznych i zawodowych całkiem dobrze i wyraża zadowolenie z powodu podjęcia decyzji o wyjeździe. $Z$ tego punktu widzenia problematyka migracji jest bardzo ważna i aktualna. Kiedy ludzie migrują z jednego narodu czy kultury do odmiennych, ich tożsamość kulturowa oraz ich poczucie przynależności podlega zmianom. Celem artykułu jest odpowiedź na pytanie: W jakim stopniu środowisko społeczno-kulturowe różnicuje dystans społeczny wobec obcych emigrantów polskich w Niemczech, ludności miejscowej oraz Polaków zamieszkujących w kraju? W prezentowanym artykule poruszone zostały różnice dotyczące dystansu społecznego badanych wobec osób innych narodowości oraz wobec wyznawców innych religii.

Słowa kluczowe: dystans społeczny, obcy, wielokulturowość, migracja zarobkowa, inne narodowości, wyznawcy innych religii

\section{Einführung}

Das Phänomen der Migration hat aufgrund seiner komplexen Prozesse ein vielseitiges Feld für die wissenschaftliche Durchdringung. Aus dem Lateinischen bedeutet Migration Wanderung bzw. migrare: wandern. Migration beschreibt grundsätzlich alle unfreiwilligen und freiwilligen Wanderungen vom Menschen ${ }^{1}$. Im Deutschen wird dieser Begriff als Umzug oder Auszug bezeichnet. Schubert \& Klein (2006) definieren Migration in der Soziologie

1 http://www.demokratiezentrum.org/fileadmin/media/pdf/wissen_migration_ begriffe.pdf (24.09.2018). 
als alle Formen der räumlichen Mobilisierung von Menschen (religiös, ethnisch) oder ethnischen Gruppen usw.

Ingrid Oswald (2007) definiert Emigration als einen Prozess der räumlichen Bewegung vom einen Ort zum anderen Ort, der mit sozialen, politischen und kulturellen Erfahrungen verbunden ist. Diese Definition versucht die Komplexität von Emigrationsprozessen zu erfassen, die mehr als eine sichtbare Bewegung von Personen um ihre Adresse oder ihre Herkunft zu ändern ist.

Der E. Lee-Ansatz macht auf die beiden Faktoren "Push" und "Pull" aufmerksam. Neoklassische Push-Pull-Modelle sehen die Auswanderung als Ergebnis einer individuellen Betrachtung von persönlichen Kosten und Nutzen (Bähr 1995, S. 399). Sie dienen dazu, die für die Auswanderung wesentlichen Bedingungen zu bestimmen (Oswald 2007, S. 71). Um die Ursachen der Emigrationsbewegungen zu illustrieren, entwickelte S. Lee Everett in den 1960er Jahren die Theorie der Push - und Pull - Faktoren, die auch unter dem Begriff der "Theorie der Emigration" bekannt ist. Dieses Modell zielt darauf ab, Probleme im Zusammenhang mit nationaler und internationaler Emigration zu lösen.

„Der Push-Faktor verursacht als abstoßender Faktor (schlechte Lebens-, Arbeits - und Wirtschaftsbedingungen) Abwanderung vom Land in die Stadt. Zudem ersetzt die zunehmende Mechanisierung der Landwirtschat die menschliche Arbeitskraft durch Maschinen wie Traktoren, Mähdrescher etc. Die Folge sind steigende Arbeitslosigkeit und Abwanderung in die Stadt, um hier einen Arbeitsplatz zu finden.

Der Pull-Faktor bewirkt als anziehende Kraft (gute Lebens-, Wirtschafts - und Arbeitsbedingungen) Zuwanderung in die Stadt. Eine Verstärkung dieser Zuwanderung wird durch Agglomerationsvorteile der Stadt verursacht - gerade diese Vorteile sind die stärksten Pull-Faktoren“2.

Das ursprüngliche Modell von Sjaastad (1962) betonte, dass Migrationen eine Antwort auf das Lohngefälle seien und zu einem Gleichgewicht zwischen Auswanderung und Einwanderung führten. In diesem Fall werden Migrationen als individuelle Investitionen in Humankapital interpretiert, was bedeutet, dass Bewegung sowohl mit monetären als auch mit nicht-monetären Kosten verbunden ist. $\mathrm{Zu}$ den finanziellen Kosten gehören beispielsweise Kosten für den Umzug in eine entferntere Region, und nichtmonetäre

2 http://www.yaclass.at/p/geografie-und-wirtschaftskunde/12-schulstufe/lokal-regional-global-vernetzungen-wahrnehmungen-konflikte-19275/staedte-als-lebensraeume-und-oekonomische-zentren-18873/re-50ad837d-5a69=4d5b-966d-fd138f688c1e (24.09.2018). 
Migrationskosten können sozialer Natur sein, wie der Verlust von Freunden oder Verwandten. Larry A. Sjaastad erklärt die internationale Migration als eine langfristige Investition in das Humankapital, die sich im Laufe der Zeit amortisiert. Dieser Einkommensgewinn des Migranten entsteht durch die Differenz zwischen den erwarteten Einkommen im Ziel - und Herkunftsland. Man kann hier Produktivität mit Humankapital gleichsetzen, somit geht ihr Anstieg mit einer höheren Entlohnung einher. Dieser Einkommensgewinn wird dann durch die Mobilitätskosten wie z.B. Transportkosten vom Herkunfts - zum Zielort, die Unterkunftskosten am Zielort reduziert.

Der Hauptvertreter der dualen Arbeitsmarkttheorie, der die beiden Arten von Marktstrukturen beschreibt, ist der Ökonom Michael J. Piore (1979). „Der Arbeitsmarkt im Empfängerland wird zum einen in einen sicheren primären kapitalintensiven Primärsektor für qualifizierte Tätigkeiten mit guter Bezahlung und guten Arbeitsbedingungen (beispielsweise Gewerkschaftsorganisation, Abfindung bei Entlassung) und zum anderen in einen instabilen arbeitsintensiven Sekundärsektor für gering qualifizierte beziehungsweise ungelernte Tätigkeiten mit niedrigen Löhnen und schlechten Arbeitsbedingungen (beispielsweise Beschäftigungsunsicherheit) segmentiert" (Vgl. Pflugbeil. Stephan Dirk (2005), S. 29 f.). Da einheimische Beschäftigte das sekundäre Segment des Arbeitsmarktes meiden, liegt es am unteren Ende der Stellenhierarchie und führt zu einem Mangel an Arbeitskräften. Weil Auswanderer um sich selbst erhalten zu können im Ausland Jobs suchen, sind sie sogar bereit, diese schlechten Bedingungen im sekundären Arbeitsmarktsegment zu akzeptieren. Für die Hauptmotivation einer Auswanderung von Menschen nach Piore (1979) sind nicht die Löhne, sondern der soziale Status verantwortlich. Das Ausführen eines Jobs unter einem qualifizierenden oder belastenden Level erschwert die Verbesserung dieses Status. Aus diesem Grund werden Arbeiten aus der unteren Hierarchieebene des Arbeitsmarktes und solche, die keine Chancen für eine spätere Karriere bieten, vermieden. Für diesen untersten Teil des Arbeitsmarktes gibt es einen unlösbaren Arbeitskräftemangel. Piore hat eine These aufgestellt, die den neoklassischen Annahmen völlig entgegengesetzt ist: „(I)t is the employers, not the workers, and the jobs, not the incomes, that are strategic" (Piore, 1979, s.19).

Eine andere Theorie, die wie Piores Theorie dem neoklassischen Modell stark widerspricht, ist Harvard Oded Starks Ökonomietheorie: The New Economics of Migration. Diese Theorie wurde in den frühen 1980er Jahren erstellt. Sie basiert auf Studien zur Land-Stadt-Migration. Generell stellt sie fest, dass Arbeitsmigration nicht als geschlechtsspezifisches Lohngefälle 
zwischen zwei Sektoren oder Ländern interpretiert werden kann, sondern als Faktoren wie Unsicherheit, Verarmung, Familienmodernisierung oder Risikoreduktion.

Deutschland ist bei weitem das begehrteste Land für Expatriates. Die Auswanderung von Polen nach Deutschland ist kein neues Phänomen. Unabhängig davon, ob polnische Bürger auswandern wollten oder mussten, hat die Emigration ihre Wurzeln im 19. Jahrhundert und über viele Jahre gewann sie neue Dimensionen und Bedeutungen.

Deutschland, als ein attraktives Reiseziel, hat seinen Arbeitsmarkt für polnische Arbeitnehmer erst 2011 geöffnet. Das Land wendet sich gegen alle Wirtschaftskrisen der letzten Jahre und ist geografisch nicht so weit von Polen entfernt wie z.B. Großbritannien oder Irland. Oft braucht man nur wenige Stunden mit dem Auto, um die deutsche Grenze zu erreichen. Aufgrund dieser räumlichen Nähe fühlen sich viele Polen in Deutschland sicherer als anderswo in Europa. Deutschland ist für Polen seit dem Brexit noch attraktiver geworden.

\section{Metodologie}

Gegenstand dieser Untersuchung war die soziale Distanz von in Deutschland lebenden polnischen Emigranten zu Menschen anderer Nationalitäten und Gläubigen einer anderen Religion, die im Kontext sozio-kultureller Bedingungen betrachtet wurden. Um die Rolle dieser Determinanten bei der Gestaltung der sozialen Distanz zu bestimmen, wurden die Einstellungen von Menschen, die nach Deutschland eingewandert sind, die Einstellungen der Menschen vor Ort und das Verhalten der Polen im Land verglichen. Es wurde daher bestimmt, inwieweit die Veränderung des sozio-kulturellen Umfelds Veränderungen in diesen Einstellungen bestimmt. Ziel der durchgeführten Forschung wurde es, die Unterschiede zwischen der Distanz von Auswanderern zu Menschen anderer Nationalität und Anhängern einer anderen Religion und den Einstellungen der im Land lebenden Deutschen und Polen in Polen zu ermitteln.

Dieses Thema scheint im Zeitalter der Massenmigration und der anhaltenden lebhaften sozialen Debatte über die Durchführung der Migrationspolitik in den Ländern der Europäischen Union besonders wichtig und aktuell zu sein.

Um eine Antwort auf die Forschungsfrage - „In welchem Maße diversifiziert das soziokulturelle Umfeld die soziale Distanz zu ausländischen polnischen Emigranten in Deutschland, der lokalen Bevölkerung und den Polen, die im 
Land leben? - zu erhalten, wurde eine Recherche in Niedersachsen-Deutschland und in Wielkopolska-Polen durchgeführt. Befragungsfragebögen (anonym) wurden den Befragten persönlich ausgehändigt. Die Anwesenheit des Forschers erlaubte es, Unklarheiten im Zusammenhang mit dem Verlauf des Befragungsgesprächs zu klären. Die ausgefüllten Fragebögen wurden direkt an die Interviewperson zurückgegeben. Die Befragten waren völlig willkürlich ausgesucht (Menschen auf der Straße, Leute, die den Laden verlassen, Studenten nach dem Vortrag). Die Auswahl der Probe war absichtlich und zufällig.

An der Studie nahmen 600 Personen mit unterschiedlichem Hintergrund teil. Ein Drittel der Befragten waren polnische Emigranten in Deutschland (33\%), ein Drittel Polen im Land (33\%) und ein Drittel die lokale Bevölkerung - in Deutschland (33\%).

Nach dem Beitritt Polens zur Europäischen Union wurden viele grenzüberschreitende Verbindungen von der Weichsel zu den Ländern der Gemeinschaft eingeführt. Diese dynamischen Prozesse haben zu einer wachsenden und sichtbaren Migration aus Polen, sowohl städtisch als auch ländlich, westlich und östlich geführt. Es wird berichtet, dass derzeit über 2,5 Millionen Polen im Exil nach Arbeit außerhalb ihres Heimatlandes suchen. Polnische Bürger gehören zu den größten Einwanderergruppen mit Wohnsitz in Deutschland. Paweł Kaczmarczyk hat bereits 2008 Informationen über Millionen von Menschen gesammelt, die ihr Land auf der Suche nach einem besseren Leben verlassen oder vor der Realität, in der sie leben fliehen. (P. Kaczmarczyk, 2008).

Die Forschung bezieht sich auf die Referenzen von Polen, die beschlossen haben, zu anderen Bürgern der Welt also zu "Fremden" auszuwandern. Um die Dynamik der Analyse zu erhalten, wurde die soziale Distanz der polnischen Emigranten vor dem Hintergrund zweier Vergleichsgruppen analysiert: Polen im Land und Deutschen in Deutschland. Hauptziel dieser Untersuchung war es, sich mit den Schwierigkeiten im Exil vertraut zu machen und die soziale Distanz der polnischen Emigranten zu "Fremden" bzw. "Anderen" zu bestimmen.

\section{Die soziale Distanz zu Menschen anderer Nationalitäten}

Die Frage der Distanz zu "Fremden" oder "Anderen" ist besonders in einer multikulturellen Gesellschaft sehr wichtig. In der Untersuchung wurden ebenfalls die demografischen und sozialen Faktoren der Befragten als intermediäre Variablen berücksichtigt. Bei der Gestaltung der Forschung wurde das Bo- 
gardus-Konzept übernommen, mit dem die Nähe und Distanz zwischen den Menschen und den damit verbundenen Erfahrungen bestimmt werden konnte.

Die in Deutschland lebenden Polen, die lokale Bevölkerung und die Polen im Land wurden in die vergleichende Analyse der sozialen Distanz gegenüber Personen amerikanischer, englischer, türkischer, jüdischer, syrischer, nigerianischer, russischer, chinesischer, deutscher und polnischer Nationalitäten einbezogen. Die Grundgruppe stellten polnischen Emigranten, die in Deutschland leben dar, die erste Vergleichsgruppe Polen im Land und die zweite Vergleichsgruppe die lokale deutsche Bevölkerung.

Das soziokulturelle Umfeld unterscheidet signifikant die soziale Distanz der polnischen Emigranten gegenüber "Fremden". Diese Unterschiede betreffen insbesondere Einstellungen gegenüber Menschen anderer Nationalitäten und Anhänger einer anderen Religion. Wie aus den durchgeführten Untersuchungen hervorgeht, zeigen polnische Auswanderer generell eine positive oder neutrale Einstellung gegenüber Menschen anderer Nationalitäten. Am positivsten ist ihre Einstellung gegenüber Deutschen, Landsleuten, Amerikanern und Engländern. Neutrale Einstellungen zeigen sich bei Juden, Chinesen, Türken, Syrern, Nigerianern und Russen.

Diagramm 1. Die soziale Distanz der polnischen Emigranten zu Menschen anderer Nationalität

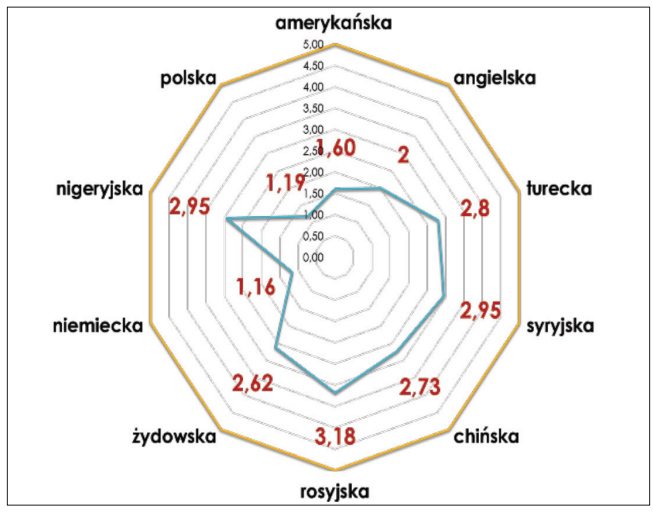

Quelle: eigene Recherchen (polnischen emigranten).

Wie die Forschungsprobleme zeigen, prägt das sozio-kulturelle Umfeld die Einstellungen von Offenheit, Toleranz und Akzeptanz von Fremden in einem größeren Ausmaß als die monokulturelle Umgebung. Die Veränderung der Umwelt trägt somit zu erheblichen positiven Veränderungen in diesem Bereich bei. 
Diagramm 2. Die soziale Distanz der Polen im Land zu Menschen anderer Nationalität

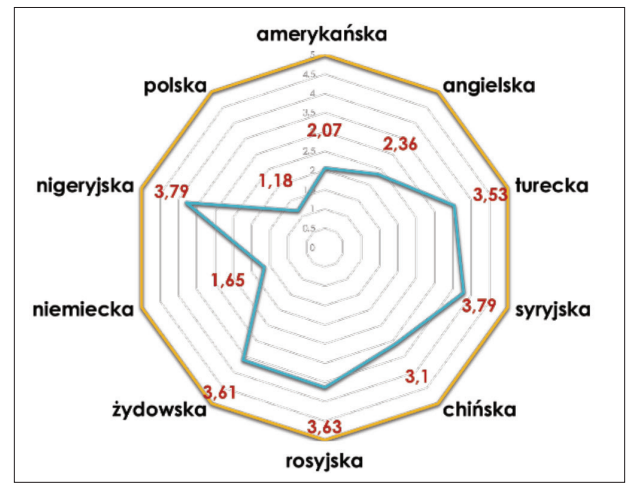

Quelle: eigene Recherchen (polnischen emigranten).

Polen, die im Land leben, erklären ziemlich traditionelle Einstellungen, die durch einen starken Mangel an Toleranz gegenüber Menschen anderer Nationen gekennzeichnet sind. Während sie eine polnische oder deutsche Nation akzeptieren können, beziehen sie sich auf die syrischen, nigerianischen und russischen Nationen mit einer sehr großen Distanz. Polen im Land betonen ihre Modernität und Fortschrittlichkeit in Bezug auf Lebensstil und Offenheit für die Welt. Das Wichtigste für sie ist der Glaube, die Kirche und der Nationalstolz. Die größte Distanz trennt die im Land lebenden Polen von syrischen und nigerianischen Staatsangehörigen und weist ihnen solche charakteristischen Merkmale und Verhaltensweisen wie Terrorismus, Brutalität und Rücksichtslosigkeit zu, die das soziale Leben bedrohen können.

Diagramm 3. Die soziale Distanz der Deutschen im Land zu Menschen anderer Nationalität

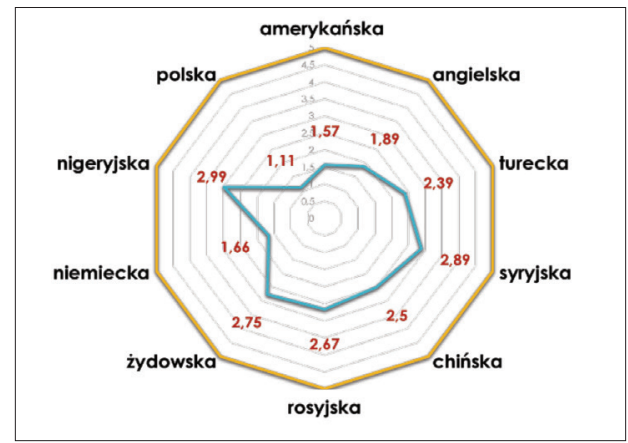

Quelle: eigene Recherchen (Deutschen in Deutschland) 
Die lokale Bevölkerung ist trotz ihrer Verbundenheit mit ihren reichen Traditionen viel offener für Menschen anderer Nationalitäten. Deutschland ist ein multikulturelles Land, das es ihnen erleichtert, verschiedene Beziehungen mit Einwanderern einzugehen, ihre Kultur und Bräuche kennenzulernen und Ängste und Vorurteile abzubauen. Deutschen haben generell eine positive Einstellung gegenüber polnischen Emigranten. Die in Deutschland lebenden Polen können sich - ihrer Meinung nach - so gut in ihre multikulturelle Umgebung integrieren, dass sie unsichtbar werden und deshalb als Chamäleons genannt werden.

Leider, so die Deutschen, sind die Polen durch mangelnde Sprachkenntnisse gekennzeichnet, dafür wird ihre sehr gute Ausbildung sehr geschätzt. Der größte Teil der Deutschen verbindet Polen mit der Fähigkeit, hart zu arbeiten und drückt großen Respekt vor schönen polnischen Frauen aus, die sich um ihr Aussehen sorgen. Die größte Distanz trennt die lokale Bevölkerung von syrischen und nigerianischen Nationalitäten. Der Grund dafür sind die extreme, Emotionen und die terroristischen Aktivitäten nicht nur in Deutschland, sondern auch weltweit.

\section{Zusammenfassung, Nationalitäten}

Um die soziale Distanz der befragten polnischen Emigranten genauer analysieren zu können, wurde ein gruppeninterner Vergleich mit demografischen Variablen durchgeführt.

Die vergleichende Analyse nach Geschlecht zeigt, dass das Geschlecht die Einstellung gegenüber allen berücksichtigten Nationalitäten signifikant unterscheidet. Generell lässt sich feststellen, dass Frauen sich von Menschen unterschiedlicher Nationalität mehr distanzieren als Männer. In Bezug auf die berufliche Situation der Befragten, hat dies keine signifikanten Auswirkungen auf die Forschungsergebnisse. Das ist interessant, da beiden Gruppierungen polnischen Einwanderer: einerseits diejenigen, die mit ihrer Ausbildung unvereinbar arbeiten und keine dauerhafte Beschäftigung haben und andererseits diejenigen, die mit dauerhafter Beschäftigung und Arbeit in Übereinstimmung tätig sind, zeigten statistisch nicht signifikanten Unterschied in den angegebenen Reaktionen. Es gibt jedoch unterschiedliche Antworten auf die soziale Distanz zwischen Menschen mit unterschiedlicher Bildung. Eine Zunahme der Distanz zwischen Befragten mit Grundschulbildung ist im Vergleich zu Personen mit höherer Bildung bemerkbar. Die Aufenthaltsdauer in Deutschland spielt auch eine wesentliche Rolle für die Identitätsbestimmung und zugleich 
für die Einstellung gegenüber "Fremden". Je kürzer die Befragten in Deutschland lebten, umso größer war die Distanz, die sie zu den Menschen anderer Nationalitäten erklärten und desto häufiger fühlten sie sich polnisch.

Die Beziehung zwischen sprachlicher Fähigkeit und sozialer Distanz war ziemlich signifikant. Basierend auf den Forschungsergebnissen wurde festgestellt, dass Sprache eine wichtige Rolle bei der Identifikation und dem Identitäts-Prozess spielt. Die Sprache eines Menschen spielt eine wichtige Rolle für seine Identifikation/Identität. Schon seit den 80er Jahren führt man in der Fremdsprachenforschung Untersuchungen zur Interdependenz zwischen Zweitsprachen-erwerb/Bilingualismus und Identität durch (Gumperz 1982; Heller 1987; Norton 2000). Man ist dabei zu dem Schluss gekommen, dass mit einer neuen Sprache eine neue (Teil-)Identität erworben wird. Mit anderen Worten: „Ein Mensch lernt eine neue Sprache und erhält dadurch, wie wir sagen, eine neue Seele. Er versetzt sich in die Haltung jener, die diese Sprache verwenden. (...) In diesem Sinn wird er zu einem anderen Menschen" (Mead 1973, 330f.)

Die Analyse des Indikators "Ort des aktuellen Wohnsitzes" hat ergeben, dass die Menschen, die in Städten mit mehr als hunderttausend Einwohner leben, weniger ethnische Vorurteile zeigen als Menschen aus Kleinstädten unter 20.000 Einwohner. Der amerikanische Anthropologe Edward T. Hall (1914-2009) untersuchte das Phänomen der Nähe und Distanz. Es setzte voraus, dass unser Gefühl von Nähe und Distanz biologisch bestimmt ist, wobei die kulturellen Einflüsse eine fundamentale Rolle spielen. Je mehr Menschen im öffentlichen Raum sind, desto schwieriger ist dieses Vorhaben. Der Unterschied zwischen einer Stadt und einem Dorf kann auch wie folgt beschrieben werden: In der Stadt sind viele Ausländer gezwungen, sich in einem geschlossenen Raum zu nähern. Aus psychologischer Sicht wird die persönliche Distanz, die wir als Schutzgebiet wahrnehmen, unfreiwillig unterbrochen (Hall, 1976).

\section{Die soziale Distanz zu Menschen anderer Religionen}

Religionen, die bei der Untersuchung der sozialen Distanz polnischer Auswanderer in Deutschland berücksichtigt wurden sind Katholizismus, Orthodoxie, Judentum, Protestantismus, Islam und Buddhismus. Die Basisgruppe besteht aus polnischen Emigranten, die in Deutschland leben, während die erste Vergleichsgruppe stellen Polen im Land und die zweite Vergleichsgruppe die lokale Bevölkerung dar. 
Aufgrund der Ergebnisse der Studie ist festzustellen, dass polnische Auswanderer, die in Deutschland bleiben, die positivsten Einstellungen vor allem gegenüber der protestantischen Konfession zeigen. Eine etwas größere Distanz wird gegenüber Katholiken wahrgenommen, obwohl die polnischen Auswanderer aus einem Land mit so tief verwurzeltem katholischen Glauben kommen. Sehr positiv bewerten polnische Emigranten in der protestantischen Religion die Gründung von Familien durch Priester, Frauen als Vikare, universelle Konfession und die Zulässigkeit von Scheidungen.

Diagramm 4. Die soziale Distanz der polnischen Emigranten gegenüber den Anhängern einer anderen Religion

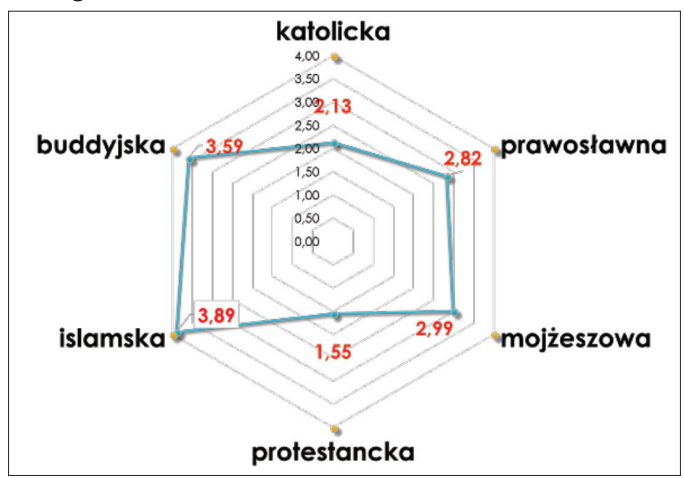

Quelle: eigene Recherchen (polnischen Emigranten).

Diagramm 5. Die soziale Distanz der Polen im Land gegenüber den Anhängern einer anderen Religion

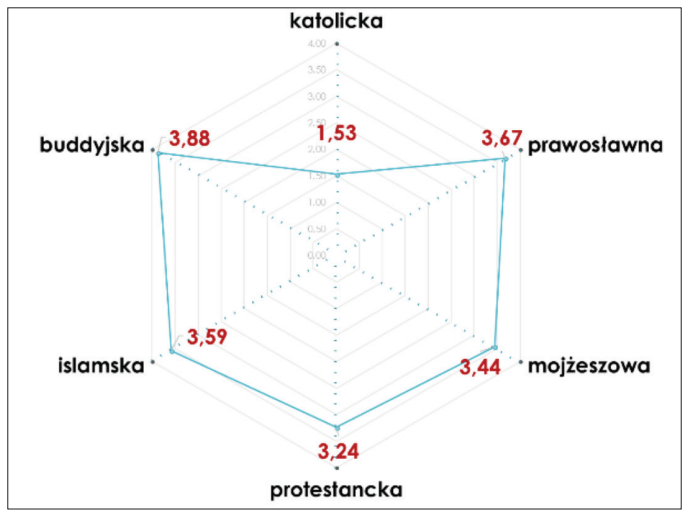

Quelle: eigene Recherchen (Polen in Polen). 
Polnische Auswanderer zeigen signifikante Vorurteile gegenüber der islamischen Religion. Kontakte zu den Anhängern des Islam hat die Hälfte der Befragten mit neutralen Emotionen charakterisiert und jede zweite Person ist nicht bereit, enge Beziehungen zu Vertretern dieser Religion zu knüpfen.

Die im Land lebenden Polen identifizierten sich am positivsten mit den Anhängern der katholischen Religion. Über zwei Drittel der Polen nehmen die katholische Religion positiv wahr, weil sie der Erziehung eines modernen Menschen dient, die der Welt gegenüber offen und tolerant gegenüber anderen Menschen ist. Fast jeder zweite Pole kann sich eine enge Beziehung zum Katholizismus vorstellen und jede zweite Person sieht Möglichkeiten zur Zusammenarbeit mit Katholiken.

Die größte Distanz trennt die Polen von den Anhängern des Judentums, wobei sich jede zweite Person keine engen Beziehungen zu Vertretern dieser Religion vorstellen kann. Die im Land lebenden Polen erklären gegenüber anderen Religionen, mit Ausnahme des Katholizismus, eine sehr hohe soziale Distanz. Eine sehr große Distanz trennt die Polen im Lande von den Anhängern der buddhistischen Religion. Ihrer Ansicht nach wird Sünde im Buddhismus gewöhnlich als Ignoranz verstanden und deshalb ist diese Religion für ein menschliches Wesen am unfreundlichsten. „Der Buddhismus nimmt an, dass die Welt der Leiden ein Ort ist, aus dem man fliehen kann und soll. Dies muss geschehen, indem man allen Bedürfnissen der Welt entsagt. Weder Freud noch Leid soll die Menschen kümmern. Sie müssen der Welt völlig entsagen, um als Erleuchtete ins Nirwana aufzusteigen. Damit entsagt man sich der Welt von den Grundlagen her. Das Weltgeschehen ist unwichtig. Nur die eigene Erleuchtung ist von Bedeutung. Eine soziale Politik hat bei einer solchen Anschauung keine Chance. Und Diktatoren werden nicht bekämpft, da auch das ein Festhalten an der Welt ist, welche man doch eigentlich für immer verlassen möchte“" ${ }^{\text {"3 }}$ Nur ein kleiner Teil dieser Polen erkennt und praktiziert die Meditation.

Die lokale Bevölkerung ist trotz ihrer Verbundenheit mit ihren reichen Traditionen sehr offen für andere Nationen und bereit, verschiedene Religionen und ihre Anhänger zu treffen. Diese Toleranz ergibt sich aus der $\mathrm{Zu}$ gehörigkeit zur multikulturellen Gesellschaft. Sie sind es, die verschiedene Beziehungen zu Einwanderern eingehen, etwas über ihre Kultur und Bräuche erfahren und so ihre Ängste und Vorurteile aufgeben.

3 http://forum.freidenkerin.at/forum/attachment.php?attachmentid=84 (24.09.2018). 
Es wurde auch festgestellt, dass die soziale Distanz der Deutschen zu den Anhängern der islamischen Religion zunimmt und negativ ist. Die Quelle dieser Einstellung können politische Erfahrungen, feststehende Stereotypen und Vorurteile sein.

Unter Berücksichtigung der Forschungsprobleme wurde festgestellt, dass die Veränderung des soziokulturellen Umfelds einen sehr bedeutenden Einfluss auf die Veränderung der Einstellung der Menschen gegenüber "Fremden" und "Anderen" hat. Polnische Emigranten sind offen gegenüber den Anhängern anderer Religionen, sie zeigen eine viel größere Toleranz als Polen, die im Land leben.

Diagramm 6. Die soziale Distanz der Deutschen im Land gegenüber den Anhängern einer anderen Religion

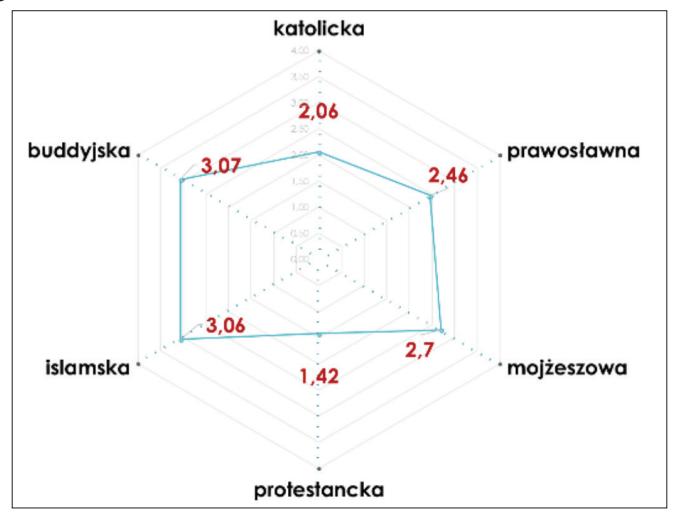

Quelle: eigene Recherchen (Deutschen in Deutschland).

Die Polen, die im Land leben, zeigen eine eher traditionelle Haltung in ihren Ansichten. Sie sind in einer Auseinandersetzung mit einer anderen Kultur, mit ihren Werten, Bräuchen und Verhaltensmustern sowie Religion und wollen ihre eigene Identität um jeden Preis behalten. Gleichzeitig sind Polen voller Ängste, die sie davon abhalten, "Fremde" oder "Andere" kennen zu lernen. Stereotype, die die Anhänger einer anderen Religion beschreiben, werden sehr leicht unter ihnen verbreitet.

\section{Zusammenfassung, Religionen}

Es stellt sich heraus, dass Polen, die in Deutschland bleiben, auch gegenüber den Anhängern anderer Religionen toleranter sind als die Polen, die in Polen 
leben. Sie stellen eine positive Einstellung gegenüber dem Protestantismus und dem Katholizismus dar, wobei die Anhänger der protestantischen Religion die positivsten sind und die Anhängern der katholischen Religion etwas weiter entfernt sind. Sehr positiv bewerten polnische Emigranten in der protestantischen Religion die Gründung von Familien durch Priester, Frauen als Vikare, universelle Konfession und die Zulässigkeit von Scheidungen. Sowohl polnische Emigranten als auch im Land lebende Polen weisen eine sehr geringe Distanz zu Katholiken auf. Doch die polnischen Emigranten sind ihnen gegenüber kritischer, sie sehen Dissonanzen zwischen Glaubensbekenntnissen und tatsächlichem Verhalten im Alltag. Die katholische Kirche in Polen wird ihrer Meinung nach immer mehr national. Polnische Emigranten beurteilen sehr negativ, dass sich die Kirche ständig bereichert, was ein häufiges Austreten aus der Kirche in Deutschland zur Folge hat. Grund ist die Vermeidung Steuern zu zahlen und die Tatsache, dass der Priester keine Familie gründen darf. Tadeusz Rydzyk und sein Medienimperium beurteilten 87 polnischen Emigranten negativ. Mariola Boensch (2013) erzielte in Bezug auf die katholische Kirche schon im Jahr 2013 ähnliche Ergebnisse. Sie bestätigte, dass junge Polen immer noch ein hohes Glaubensniveau verkünden würden, sich aber von der Glaubenspraxis und der institutionellen Kirche distanzieren möchten. Boensch betonte, dass sie sowohl die Lehren der Kirche als auch die überholte Haltung in praktischen Lebensfragen kritisieren. Gleichzeitig steigt die Akzeptanz von Sex ohne Ehe, Scheidung und Homosexualität. Laut Mechtenberg ist die Situation der katholischen Kirche in Polen immer noch schlecht. Auf der einen Seite wird das Kirchenbild gewonnen, das sehr stark national und traditionell geprägt ist und auch politisch der Nationalen Konservativen Partei der PiS ähnlich ist (Mechtenberg Theo, 2013). Zu den Feinden der Kirche zählt Mechtenberg den Radiosender von Pater Rydzyk "Radio Maryja“, sein Medienimperium, das TV-Programm und Publikationen wie z.B. "Gazeta Wyborcza“ (Theo Mechtenberg, 2013). Gleichzeitig wird der "offene Katholizismus" gefördert, der insbesondere von katholischen Intellektuellen Kreisen vertreten wird. Diese Art des Katholizismus möchte die demokratische und pluralistische Form der neuen polnischen Gesellschaft akzeptieren und ist bereit für einen Wandel, für Kompromisse und sucht einen Dialog mit den Dissidenten (Mechtenberg Theo, 2013, S. 5). Die größte soziale Distanz besteht zwischen polnischen Emigranten und Menschen islamischer Religion. Muslime glauben an das ewige Leben nach dem Tod und diejenigen, die die Gebote Gottes befolgen, können auf das Paradies zählen. Polnische Emigranten bewerten die Regeln des Koran, 
die ein legales Bild für den Alltag der islamischen Gesellschaft darstellen, negativ. Der Islam ist jedoch das gesamte Rechtssystem, das in jeden Bereich des Lebens durchdringt. Eine Person, die ein Muslim ist, kann nicht selbstständig aus den Glauben austreten und wenn die Person sich vom Glauben zurückzieht, wird ihr der Tod angedroht. „Die Botschaft von Allah ist der Koran. Er enthält die Lehre des Islam. Muslime glauben, dass der Erzengel Gabriel sie dem Propheten Mohammed verkündet hat. Mohammed hat die Botschaft Allahs dann an die Menschen weitergegeben. Danach möchte Allah, dass die Menschen an ihn glauben, dass sie freundlich zueinander sind, dass sie sich gegenseitig helfen und friedlich miteinander leben. Damit das klappt, enthält der Koran auch Anweisungen, wie die Menschen miteinander umgehen und wie sie leben sollen"4.

Die im Land lebenden Polen erklären gegenüber anderen Religionen, mit Ausnahme des Katholizismus, eine sehr hohe soziale Distanz wobei die größte Distanz trennt die Polen im Lande von den Anhängern der buddhistischen Religion. Ihrer Ansicht nach wird Sünde im Buddhismus gewöhnlich als Ignoranz verstanden und deshalb ist diese Religion für ein menschliches Wesen am unfreundlichsten. Nur ein kleiner Teil dieser Polen erkennt Meditation. In Bezug auf die katholische Religion glauben die Polen im Land, dass das Schicksal für immer mit Gott sein soll. Die heilige Kommunion und das Bekenntnis sind für sie gleichermaßen wichtig und die sonntägliche Teilnahme an der Messe ist obligatorisch. Homosexualität und In-vitro-Befruchtung ist für Katholiken eine große Sünde. Abtreibung ist und bleibt ein Mord. Die lokale Bevölkerung bekennt sich gegenüber den Anhängern der protestantischen Religion eindeutig positiv. Die größte Distanz trennt die Deutschen von den Anhängern islamischer und buddhistischer Religionen. Die multikulturelle Gesellschaft, die Deutschland ist, hat gelernt die Vielfalt von Nationalitäten zu respektieren und die Religion ihrer Vorfahren zu pflegen. Die protestantische Religion ist laut der örtlichen Bevölkerung neben Katholizismus und Orthodoxie einer der Hauptzweige des Christentums. Diese Konfessionen entstanden als Folge der reformatorischen Bewegungen innerhalb der römisch-katholischen Kirche, die im 16. Jahrhundert mit der Aufführung von Martin Luther begann. Größere Distanz trennt die Deutschen von den Anhängern islamischer und buddhistischer Religionen. Der Buddhismus wird von den Einheimischen als Seelenwanderung oder Reinkarnation definiert. Buddhismus scheint für sie als eine Religion mit ihren

4 https://www.religionen-entdecken.de/lexikon/a/allah (24.09.2018). 
Geboten und Verboten recht kompliziert zu sein. Der Islam verbindet die lokale Bevölkerung mit dem Koran, mit Gewalt und mit Allah. Deutsche äußern sich sehr negativ über die Beschneidung von Jungen, über die Bestattung von Muslimen an einem Tag ohne Sarg und in Richtung Mekka, und dass viele muslimischen Mädchen keine ausreichende Ausbildung besitzen und gezwungen werden ein Kopftuch zu tragen.

\section{Schlusswort}

Seit Polens Beitritt zur Europäischen Union im Jahr 2004 kann jeder Pole im Ausland studieren, Sprachen lernen und Karriere machen. Bewegung ist ein natürliches soziales Phänomen, aber nur, wenn es kontrolliert wird. Die von Infratest dimap durchgeführte Umfrage zeigt, dass in Vorurteilen nur wenige Ausländer leben. Deutsche Forscher sind überzeugt, dass diese über 50-jährige Analyse dazu beitragen kann, die aktuelle Situation von Ausländern zu meistern. Der Mangel an Sicherheit im Umgang mit Flüchtlingen ist besonders hoch für Personen, die keine Erfahrung mit Einwanderung haben. Forscher erklären, dass sich die Menschen besonders für die "Unbekannten" oder "Fremden" interessieren. Ulrich Wagner, Professor für Sozialpsychologie an der Universität Marburg und der Initiator des Briefes an die Bundesregierung erklärt, dass die Hypothese des interkulturellen Kontakts immer noch sehr aktuell ist. Ein flüchtiges Treffen auf der Straße reicht nicht aus, um sie zu verurteilen. Der Vorschlag von J. Wirz (1997) beschäftigt sich nicht damit, ob wir andere Kulturen verstehen können, sondern ob wir unseren Nächsten verstehen können. Kommunikation mit Angehörigen anderer Nationalitäten ist keine Kommunikation mit diesen Nationalitäten, sondern vor allem mit Menschen. Es gibt keine nationalistischen Verbindungen in Geschäftsbeziehungen, aber Menschen, die sie führen. Die Praxis des Lebens, die wir jeden Tag miteinander teilen („transkulturell“ oder „interkulturell“) ist ein kontinuierlicher Prozess und schafft Gemeinsamkeiten und Unterschiede viel früher - als wir uns bewusst sind - nämlich schon zwischen Mutter und Kind (Wirz J., 1997). Migration macht den "Horizont der Gefühle", wie Sehnsucht, Verlust, Traurigkeit intensiver. Die Anpassung in einem neuen Land ist ein langer Prozess, der niemals enden wird. In diesem Prozess erkennen MigrantInnen ihre Stärken und sind gezwungen, sich selbst und die neue Lebenssituation zu erkennen und zu akzeptieren. Dank dieser Akzeptanz steigt auch die Chance, neue Beziehungen aufzubauen, neue Freundschaften zu schließen und neue Potenziale für ein aktives Leben zu schaffen. Dieses um- 
fassende Problem der Akkulturation und sozialen Distanz gegenüber "Fremden" ist besonders in einer multikulturellen Gesellschaft extrem wichtig. Fasst man die Ergebnisse der Forschung zusammen, können wir schließen, dass der Beitritt Polens zur Europäischen Union, für Polen uneingeschränkte Mobilität eröffnet hat. Er hat den Polen ermöglicht, über eine andere Kultur und eine andere Religion zu lernen. Gleichzeitig erleichtert er die Beseitigung von Vorurteilen und die Reduktion des Provinzialismus. Während wir in einer multikulturellen Gesellschaft bleiben, werden wir offener und die soziale Distanz zu "Anderen" oder "Fremden" wird definitiv niedriger.

\section{Bibliografie}

Babka von Gostomski, Ch. 2016. Aspekte der Integration von zugewanderter rumänischen und polnischen Staatsangehoörigen in Deutschland. Nürnberg: BAMF - Kurzanalyse, ss. 1-12.

Bähr, J. 1995. Internationale Wanderungen in Vergangenheit und Gegenwart. Geographische Rundschau. 47 (7-8), ss. 405-409.

Bera, R. 2012. Poczucie odpowiedzialności zawodowej osób migrujacych zarobkowo. Perspektywa pedagogiczna. Lublin: UMCS.

Boensch, M. 2013. Kulturelle Unterschiede in der deutsch-polnischen Wirtschaftskommunikation - Fiktion oder Realität? Eine soziokulturelle Umweltanalyse. Europa-Universität Viadrina Frankfurt: Kulturwissenschaftliche Fakultät.

Breitkreuz, R. 2009. Analyse der Push-und Pull-Faktoren der Migration. Taschenbuch. Norderstedt: Grin Verlag.

Brzozowski, P. 2007. Wzorcowa hierarchia wartości. Polska, Europejska czy Uniwersalna? Lublin: UMCS.

Burszta, W. J. 1998. Antropologia kultury. Poznań: UAM.

Cieciuch, J. 2009. Nadzieja jako moderator zwiazku poczucia koherencji $z$ preferencjami wartości. Warszawa: Referat na Sympozjum Towarzystwa Fides et ratio.

Czerwiński, M. 1976. Pojęcie stylu życia i jego implikacje. W: Siciński, A. red. Styl życia. Koncepcje i propozycje. Warszawa: PWN, ss. 33-69.

Gumperz, J.J. 1982. Discourse Strategies. Cambridge: Cambridge University Press.

Hall, T.E. 1976. Beyond Culture. New York: Anchor Books ed.

Heller, M. 1987. Language and Identity. In: U. Ammon et al. eds. Sociolingu- 
istics/Soziolinguistik. Ein internationales Handbuch zur Wissenschaft von Sprache und Gesellschaft (HKS 3.1.). Berlin: Walter de Gruyter, pp. 780-784 .

Jawłowska, A. 1976. Styl życia a wartości. W: Siciński, A. red. Styl życia. Koncepcje i propozycje. Warszawa: PWN, ss. 205-239.

Kaczmarek, G. 1986. Zróżnicowanie społeczne a styl życia - studium analityczne. Warszawa - Poznań: PWN.

Kaczmarczyk, P. 2008. Arbeitsmigration und der polnische Arbeitsmarkt. Berlin: Working Paper des Progressiven Zentrums.

Kubinowski, D. 2010. Jakościowe badania pedagogiczne. Filozofia - Metodyka - Ewaluacja. Lublin: UMCS.

Lee, E.S. 1972. Eine Theorie der Wanderung. In: Széll, G. Hrsg. Regionale Mobilität: elf Aufsätze. München: Nymphenburger Verl.-Handl, pp. 115-129. Mead, G.H. 1973. Geist, Identität und Gesellschaft aus der Sicht des Sozialbehaviorismus. Taschenbuch. Auflage 18. Berlin: Suhrkamp Verlag.

Mechtenberg, T. 2013. Interkulturelle Empathie: Beiträge zur deutsch-polnischen Verständigung (Europäische Studien zur Germanistik, Kulturwissenschaft und Linguistik. Band 4. Frankfurt am Main: Peter Lang GmbH, Internationaler Verlag der Wissenschaften.

Nikitorowicz, J. i Sobecki, M. red. 1999. Edukacja międzykulturowa w wymiarze instytucjonalnym. Białystok: Wydawnictwo Uniwersyteckie „Trans Humana".

Neubert, Stefan, Roth, Hans-Joachim, und Yildiz Erol. 2013. Multikulturalität in der Diskussion: Neuere Beiträge zu einem umstrittenen Konzept (Interkulturelle Studien). Wiesbaden: Springer VS.

Norton, B. 2000. Identity and Language Learning: Gender, Ethnicity and Educational Change. Essex: Pearson Education.

Olbrycht, K. 2004. Miejsce koncepcji człowieka w edukacji ku wartościom. W: Szerląg, A. red. Edukacja ku wartościom. Kraków: Oficyna Wydawnicza „Impuls”, ss. 61-69.

Oswald, I. 2007. Migratiossoziologie. Konstanz: UTB GmbH.

Palska, H. 1999. Styl życia. Stan badań i nowe potrzeby badawcze. W: Gawin, D. red. Homo eligens: społeczeństwo świadomego wyboru. Warszawa: Wydawnictwo IFiS PAN, ss. 53-68.

Piore, M.J. 1979. Birds of Passage: Migrant Labor and Industrial Societies. Cambridge: Cambridge University Press.

Plopa, M. 2005. Więzi w małżeństwie i rodzinie. Metody badań. Kraków: Oficyna Wydawnicza „Impuls”. 
Pflugbeil, S.D. 2005. Auswirkungen der internationalen Migration auf die Bundesrepublik Deutschland. Theoretische und empirische Analysen vor dem Hintergrund der EU-Osterweiterung. Regensburg: Wirtschaftswissenschaftliche Fakultät der Universität.

Pries, L. 2008. Transnationalisierung der sozialen Welt. Sozialräume jenseits von Nationalgesellschaften. Berlin: Suhrkamp Verlag.

Schubert, K. und Klein, M. 2006. Das Politiklexikon (4th. Edition). Bonn: Dietz.

Siebert, H. 1995. Internationale Migrationen aus wirtschaftswissenschaftlicher Sicht. Geographische Rundschau. 47 (7-8), ss. 405-409.

Simmel, G. 2006. Die Großstädte und das Geistesleben. Frankfurt am Main: Suhrkamp Verlag.

Simmel, G. 1908. Soziologie. Untersuchungen über die Formen der Vergesellschaftung. Leipzig: Duncker \& Humblot.

Sjastaad, L.A. 1962. The Costs and Returns of Human Migration. The Journal of Political Economy. 70, pp. 80-93.

Stark, O. 1991. The Migration of Labor. New York: Blackwell Pub.

Suchodolski, B. 1979. Kształt życia. Warszawa: Nasza Księgarnia.

Śliwerski, B. 1998. Wspótczesne teorie i nurty wychowania. Kraków: Oficyna Wydawnicza „Impuls”.

Todaro, M.P. 1980. Internal migration in developing countries: A survey. In: Easterlin, R.A. ed. Population and Economic Change in Developing Countries. Chicago: University of Chicago Press, pp. 361-401.

Wirz, J. 1997. The Future of DNA. Luxemburg: Springer.

\section{Social distance of Polish emigrants to Others in a new sociocultural environment}

Abstract: This work is an attempt to describe the phenomenon of the latest migration of Poles, which took place after 2004, when Poland became a member state of the European Union and when many Poles found employment abroad. The majority of migrants from Poland, relatively well-educated, young and having unpleasant experiences in the home country, manage quite well in the new social and professional circumstances and are satisfied with the decision to leave. From this point of view, migration is very important and timely. When people migrate from one nation or another to another, they take their identity with them. In a new culture, their cultural identity and their sense of belonging are subject to change. The author tries to answer the question to what extent the socio-cultural environ- 
ment diversifies the social distance towards foreign Polish emigres in Germany, the local population and Poles living in the country. The presented work discusses differences regarding the social distance of respondents towards people of other nationalities and towards followers of other religions.

Keywords: social distance, foreign, profitable migration, other nationalities, followers of other religions 